\title{
Resolving color differences of comet 41P/Tuttle-Giacobini-Kresák
}

\author{
Igor Luk'yanyk $^{1}$, Evgenij Zubko ${ }^{2}$, Gorden Videen ${ }^{2,3}$, Oleksandra Ivanova ${ }^{1,4,5}$, and Anton Kochergin ${ }^{6,7}$ \\ 1 Astronomical Observatory, Taras Shevchenko National University of Kyiv, 3 Observatorna St., 04053 Kyiv, Ukraine \\ e-mail: iluk@observ.univ.kiev.ua \\ 2 Humanitas College, Kyung Hee University, 1732, Deogyeong-daero, Giheung-gu, Yongin-si, Gyeonggi-do 17104, South Korea \\ 3 Space Science Institute, 4750 Walnut St., Suite 205, Boulder, CO 80301, USA \\ 4 Astronomical Institute of the Slovak Academy of Sciences, 05960 Tatranská Lomnica, Slovak Republic \\ 5 Main Astronomical Observatory of National Academy of Sciences, 27 Akademika Zabolotnoho St., 03143 Kyiv, Ukraine \\ ${ }^{6}$ School of Natural Sciences, Far Eastern Federal University, 8 Sukhanova St., Vladivostok 690950, Russia \\ 7 Institute of Applied Astronomy of RAS, 10 Kutuzova Emb., Saint-Petersburg 191187, Russia
}

Received 11 July 2020 / Accepted 16 September 2020

\section{ABSTRACT}

\begin{abstract}
Three different measurement campaigns have resulted in three drastically different sets of color measurements of Comet 41P/TuttleGiacobini-Kresák, ranging from a strongly red to a strongly blue color. Although the color slope is normalized to the wavelength range between the filters used, this only serves to partially normalize the resulting color, as the reflectance of cometary dust has a very strong dependence on particle absorption, which may change significantly over the wavelength range of measurement. We demonstrate that the different measurements are physical and are consistent with real materials; for example, we are able to reproduce the color measured during one epoch in which both strong blue and red color slopes were measured almost simultaneously in different filter sets with the mineral dust pyroxene. Such measurements with different filter sets serve as an additional constraint in modeling dust properties.
\end{abstract}

Key words. comets: general - comets: individual: 41P/Tuttle-Giacobini-Kresak

\section{Introduction}

Multi-wavelength photometry of Comet $41 \mathrm{P} /$ Tuttle-GiacobiniKresák's (hereafter 41P/T-G-K) 2017 apparition has been conducted by at least three independent groups (i.e., Boehnhardt et al. 2020; Luk'yanyk et al. 2019; Moulane et al. 2018). A comprehensive analysis of these observations resulted in significantly different color slopes obtained by these different groups over different epochs. This Letter aims to clarify the color-slope conception and provide an explanation for the difference between the results reported in Boehnhardt et al. (2020), Luk'yanyk et al. (2019), and Moulane et al. (2018).

The color slope $S^{\prime}$ is defined as follows (i.e., A'Hearn et al. 1984; Lamy et al. 2011):

$S^{\prime}=\frac{10^{0.4 \Delta m}-1}{10^{0.4 \Delta m}+1} \times \frac{20}{\lambda_{2}-\lambda_{1}}=\frac{A_{2}-A_{1}}{A_{2}+A_{1}} \times \frac{20}{\lambda_{2}-\lambda_{1}}$.

Here, $\Delta m$ denotes the true color index of the comet, that is reduced for the color index of the Sun; $A_{2}$ and $A_{1}$ stand for the reflectance of the comet at effective wavelengths $\lambda_{1}$ and $\lambda_{2}$ of the utilized filters $\left(\lambda_{2}<\lambda_{1}\right)$; and $\lambda$ is measured in $\mu \mathrm{m}$ and $S^{\prime}$ is measured in $\%$ per $0.1 \mu \mathrm{m}$. It is important to note that $S^{\prime}<0$ and $S^{\prime}>0$ suggest a blue and red color, respectively. Since the color slope is normalized to the wavelength difference, it is often interpreted in terms of its independence from the specific choice of the filters used. However, this is not necessarily true, as we demonstrate in this Letter.

The color index does not remain constant, but it depends on the composition of the coma, which can change, for instance, with cometary activity. Luk'yanyk et al. (2019) found a rapid and dramatic change in the color slope in the inner 41P/T-G$\mathrm{K}$ coma (projected diameter of $\sim 2000 \mathrm{~km}$ ) between March 3 and 4,2017 . They obtained color slope from photometric observations with the $V\left(\lambda_{1}=0.551 \mu \mathrm{m}\right)$ and $R\left(\lambda_{2}=0.658 \mu \mathrm{m}\right)$ filters of the Johnson-Cousins photometric system. While the inner coma revealed a blue color with $S^{\prime}=(-10.15 \pm 3.43) \%$ per $0.1 \mu \mathrm{m}$ on March 3; on the next night, March 4, it was found to be red in appearance with $S^{\prime}=(16.48 \pm 4.27) \%$ per $0.1 \mu \mathrm{m}$.

Boehnhardt et al. (2020) did not see rapid color variations in Comet 41P/T-G-K similar to what was reported in Luk'yanyk et al. (2019). However, they started their observations on March 11, 2017, a week after the phenomenon was observed by Luk' yanyk et al. (2019), and this type of activity would have changed in the subsequent period between measurements. In comparison, Boehnhardt et al. (2020) also considered the results from Moulane et al. (2018) obtained on March 3, 2017. For this epoch, Moulane et al. (2018) reported two values of the $A f \rho$ parameter (e.g., A'Hearn et al. 1984) measured with the $B C\left(\lambda_{1}=0.445 \mu \mathrm{m}\right)$ and $R C\left(\lambda_{2}=0.715 \mu \mathrm{m}\right)$ filters from the HB photometric system (Farnham et al. 2000). These data yield $S^{\prime}=(11 \pm 3) \%$ per $0.1 \mu \mathrm{m}$, which appears dramatically inconsistent with the results of Luk'yanyk et al. (2019) for nearly the same epoch, that is, $S^{\prime}=(-10 \pm 3) \%$ per $0.1 \mu \mathrm{m}$. There were, however, significant differences between the measurement conditions. Moulane et al. (2018) gathered their signal within an aperture that was a factor of 5 larger than that of Luk'yanyk et al. (2019). Furthermore, the $A f \rho$ parameter was adjusted to the exact backscattering regime, that is, phase angle $\alpha=0^{\circ}$; whereas, 
Luk'yanyk et al. (2019) considered the color slope at a phase angle of $\alpha=24.8^{\circ}$, as of March 3, 2017.

While Boehnhardtetal. (2020) suggest differences in the results of Moulane et al. (2018) with those of Luk'yanyk et al. (2019), there are also differences in the results of Boehnhardt et al. (2020) with those of Moulane et al. (2018). For instance, we adapted the measurements from Moulane et al. (2018) of the Af $\rho$ parameter in Comet 41P/T-G-K with the above-mentioned $B C$ and $R C$ filters on April 13, 2017. Using Eq. (1), we find $S^{\prime}=(18.03 \pm 5.76) \%$ per $0.1 \mu \mathrm{m}$. Less than one day earlier, on April 12, Boehnhardt et al. (2020) report the brightness of Comet 41P/T-G-K with the SDSS- $r$ $\left(\lambda_{1}=0.6303 \mu \mathrm{m}\right)$ and SDSS- $i\left(\lambda_{2}=0.7595 \mu \mathrm{m}\right)$ filters. Taking the corresponding color index of the Sun $\left(0.11^{m}\right)$ into account, we obtain the true color index $\Delta m=(-0.17 \pm 0.06)$ mag for the aperture diameter of $10000 \mathrm{~km}$, which is the same as in Moulane et al. (2018). For such a color index, Eq. (1) yields $S^{\prime}=(-12.09 \pm 4.25) \%$ per $0.1 \mu \mathrm{m}$. In other words, the color slope inferred from data reported by Boehnhardt et al. (2020) also dramatically differ from the result of Moulane et al. (2018). While all three sets of photometric observations suggest differences in the color slope, we show, in the next section, that this results from the use of different sets of filters. Furthermore, the color slope obtained with different filter sets should be considered as complementary to one another; whereas, their joint analysis provides important clues for retrieving the chemical composition of dust in Comet 41P/T-G-K.

We note that gaseous emission can contribute to the flux measured through the broadband filters; however, the signal from gaseous emission is efficiently dampened by small aperture sizes. Using the Haser model (e.g., Combi et al. 2004), neither gaseous species whose emission lines appear in cometary spectra directly evaporate from the nucleus; that is to say, every species results from the photodissociation of some parent. The $\mathrm{C}_{2}$ molecules appear from the dissociation of either $\mathrm{C}_{2} \mathrm{H}_{2}\left(\mathrm{C}_{2} \mathrm{H}_{6}\right)$ molecules (e.g., Jackson et al. 1991) or from the so-called CHON particles (e.g., Clairemidi et al. 1990; Rousselot et al. 1994). This dissociation process takes time over which the parent molecules may move a considerable distance from the nucleus. In the Haser model, this characteristic is referred to as the scale length of the $C_{2}$ parent molecules (e.g., Combi et al. 2004). The dissociation is a probabilistic process, and the scale length refers to the distance where the population of parent molecules falls to $1 / e$ of their initial number. Fink et al. (1991) suggest a scale distance of $\sim 58000 \mathrm{~km}$ for the $\mathrm{C}_{2}$ parent at a heliocentric distance of $r_{\mathrm{h}}=1$ au with an $\sim r_{\mathrm{h}}^{2}$ dependence on the heliocentric distance. At the commencement of measurements in March 2017, Comet $41 \mathrm{P} / \mathrm{T}-\mathrm{G}-\mathrm{K}$ was at $r_{\mathrm{h}}=1.17 \mathrm{au}$, which suggests that the $\mathrm{C}_{2}$-parent scale distance is $\sim 79000 \mathrm{~km}$. This implies that within the cometocentric distances of $\sim 1000 \mathrm{~km}$ used in Luk'yanyk et al. (2019), only $\sim 1.3 \%$ of the $\mathrm{C}_{2}$ progenitors dissociate (see, e.g., discussion of Eq. (5) in Zubko et al. 2020a). When considering the largest aperture used in Boehnhardt et al. (2020), that is a radius of $5000 \mathrm{~km}$, the amount of dissociated $\mathrm{C}_{2}$ parents increases to only $6.1 \%$. These factors significantly reduce the influence of gas emissions on the measured fluxes. This has been demonstrated, for instance, in direct ground-based measurements by Jewitt et al. (1982) who integrated the spectra of three different comets, both with and without emission lines over wavelengths ranging from 0.5 to $0.7 \mu \mathrm{m}$, and they found that the contamination caused by gases contributes only $\sim 1 \%$ of the measured flux. In one of those three comets, the emission lines clearly appear in its spectrum; nevertheless, the continuum in the innermost coma dominates the signal. Picazzio et al. (2019) similarly found gaseous contamination of the light-scattering response in the inner coma to be only $2.5 \%$. Interestingly, in situ measurements of the gaseous emission and continuum in Comet $1 \mathrm{P} /$ Halley (Clairemidi et al. 1990) also reveal a dramatic change in the relative contribution of the continuum and gaseous emission with distance to the nucleus. For instance, their Fig. 1 demonstrates that the flux from continuum becomes comparable to that from gaseous emissions only in the outer coma, when the line of sight is shifted from the nucleus location by $34504 \mathrm{~km}$.

In conclusion, we quantitatively estimated the impact of gaseous emission on the observations of Luk'yanyk et al. (2019) on March 3.06 and 4.00, 2017, that is to say epochs when the innermost coma of $41 \mathrm{P} / \mathrm{T}-\mathrm{G}-\mathrm{K}$ has experienced a dramatic change in color. First, using the Haser model, we computed the column density of the $C_{2}$ molecules in the $41 \mathrm{P} / \mathrm{T}-\mathrm{G}-\mathrm{K}$ coma (e.g., Eq. (4) of Combi et al. 2004). These molecules are known to be the strongest gaseous-emission contaminants of cometary spectra in the visible (e.g., Farnham et al. 2000; Ivanova et al. 2017a; Chubko et al. 2009; Rosenbush et al. 2020). In particular, their effect is the most apparent in the broadband $V$ filter of the Johnson photometric system used in Luk'yanyk et al. (2019). Computation of the column density of the $\mathrm{C}_{2}$ molecules requires their production rate $\mathrm{Q}\left(\mathrm{C}_{2}\right)$. These data are available from Moulane et al. (2018) for epochs that are very close to observations by Luk'yanyk et al. (2019). On March 03.12, 2017, $Q\left(C_{2}\right)=3.16 \times 10^{24}$ molecules per second, and on March $04.16,2017, \mathrm{Q}\left(\mathrm{C}_{2}\right)=3.78 \times 10^{24}$ molecules per second. It is important to note that we simultaneously adapted the values of the scale lengths and velocity of the parent and daughter molecules from Moulane et al. (2018) since $\mathrm{Q}\left(\mathrm{C}_{2}\right)$ is dependent on the choice of those model parameters. What emerges from our computations is a radial profile of column density of the $\mathrm{C}_{2}$ molecules in the $41 \mathrm{P} / \mathrm{T}-\mathrm{G}-\mathrm{K}$ coma. We integrated this profile within a circular aperture centered at the nucleus and having a radius of $1000 \mathrm{~km}$, and we obtain the number of $\mathrm{C}_{2}$ molecules within the field of view in the observations of Luk'yanyk et al. (2019). For the former epoch, we obtain $\mathrm{N}_{\mathrm{C}_{2}}=2.49 \times 10^{26}$ molecules, and for the later epoch, $\mathrm{N}_{\mathrm{C}_{2}}=3.00 \times 10^{26}$ molecules. Fluorescence efficiency of a single $\mathrm{C}_{2}$ molecule $(\Delta v=0)$, whose emission lines almost entirely fall within the $V$-filter passband (e.g., Farnham et al. 2000), is equal to $4.5 \times 10^{-13} \mathrm{erg} \mathrm{s}^{-1}$ at $r_{\mathrm{h}}=1 \mathrm{au}$, which is scaled to an arbitrary heliocentric distance as $r_{\mathrm{h}}^{-2}$ (A'Hearn et al. 1995). Using these data, one can compute the cumulative flux from the $\mathrm{C}_{2}$ molecules $(\Delta v=0)$ in Comet $41 \mathrm{P} / \mathrm{T}-\mathrm{G}-\mathrm{K}$ within the aperture radius of $1000 \mathrm{~km}$. We obtain $6.49 \times 10^{-13} \mathrm{erg} \mathrm{s}^{-1} \mathrm{~cm}^{-2}$ on March 3.12 and $8.77 \times 10^{-13} \mathrm{erg} \mathrm{s}^{-1} \mathrm{~cm}^{-2}$. These fluxes correspond to a brightness of $16.76 \mathrm{mag}$ and $16.43 \mathrm{mag}$ in the $V$ filter, respectively. However, on those nights, Luk'yanyk et al. (2019) found the apparent magnitude of the $41 \mathrm{P} / \mathrm{T}-\mathrm{G}-\mathrm{K}$ coma to be $V=(13.59 \pm 0.04) \mathrm{mag}$ and $(13.61 \pm 0.02) \mathrm{mag}$, respectively. We note that the brightness in the $V$ and $R$ filters was not reported in Luk'yanyk et al. (2019), only their difference. As one can see, the $\mathrm{C}_{2}$ emission appears to be a factor of 18.6 (March 3) or 13.5 (March 4) times weaker compared to the brightness of Comet $41 \mathrm{P} / \mathrm{T}-\mathrm{G}-\mathrm{K}$ in the $1000 \mathrm{~km}$ aperture. In other words, the strongest gaseous-emission contaminant contributes only $5-8 \%$ of the total flux measured in the innermost coma. It is also worth noting that the increase in the $\mathrm{C}_{2}$ emission between March 3 and 4 is accompanied with a significant reddening of the $41 \mathrm{P} / \mathrm{T}-\mathrm{G}-$ $\mathrm{K}$ coma, lending further support that the dramatic variations of the color is due to a changing dust population. Finally, we note that the filters used in Boehnhardt et al. (2020) are isolated from the $\mathrm{C}_{2}$ emission and should be even less affected by gaseous 
contamination. Therefore, the significant temporal and spatial variations of the photometric color in Comet 41P/T-G-K reported in the literature must be attributed to changes in its dust populations.

\section{Properties of the color slope}

The reflectance of dust particles in comets is dependent on their microphysical properties. Two of the most important characteristics are their size distribution and complex refractive index $m$. In principle, the morphology of dust particles also plays an important role. However, dust particles are highly irregular in shape, and the light scattering from distributions of such particles has a much lesser dependence on their specific shapes than the size distribution and the refractive index Zubko et al. (2015a). Light scattering by cometary dust is predominantly governed by submicron and micron-sized particles (e.g., A'Hearn et al. 2011; Zubko et al. 2020b). In situ studies of comets suggest a power-law size distribution of their dust particles $r^{-n}$, with the index $n$ ranging from 1.5 to 3 at $r<10 \mu \mathrm{m}$ (Mazets et al. 1986; Price et al. 2010). It is worth noting that the power index tends to increase with $r$, up to $n=4.8$ in decimeter-sized particles (Virkki et al. 2019).

Dust in comets is composed of numerous components with different chemical and mineralogical compositions. The color slope in some comets also suggests the presence of materials with a noticeable wavelength dependence on their refractive index $m$ (Zubko et al. 2015b; Ivanova et al. 2017b; Luk'yanyk et al. 2019; Chornaya et al. 2020). Laboratory measurements of the refractive index in only a few presumable analogs of cometary-dust constituent materials are available in the literature (e.g., Duley 1984; Warren 1984; Khare et al. 1990, 1993; Jenniskens 1993; Dorschner et al. 1995). These analogs could be conditionally divided into two groups, having (1) a weak and (2) strong wavelength dependence on the imaginary part of refractive index $\operatorname{Im}(m)$ in the visible. As demonstrated in Zubko et al. (2014), weak variations of the material absorption, $\Delta \operatorname{Im}(m) \leq 0.001$, do not affect the light-scattering response in micron-sized particles at the level that could be detected in practice. Examples of this first group are water ice (Warren 1984), Mg-rich silicates (Dorschner et al. 1995), and, to some extent, the ice tholin (Khare et al. 1993). The second group comprises materials whose imaginary part of the refractive index experiences significant variations in the visible, $\Delta \operatorname{Im}(m) \geq 0.01$, producing a strong effect on their light-scattering response (Zubko et al. 2014, 2015b). It is worth noting that the effect of $\Delta \operatorname{Im}(m)$ tends to decrease while the material absorption grows (Zubko et al. 2015b). For instance, a $\Delta \operatorname{Im}(m)=0.01$ strongly affects light scattering by micron-sized particles with $\operatorname{Im}(m) \sim 0$, but its effect practically disappears when $\operatorname{Im}(m) \sim 0.6$ (Zubko et al. 2014). A strong wavelength dependence of $\operatorname{Im}(m)$ in the visible appears in the $\mathrm{Mg}$-Fe silicates (Dorschner et al. 1995) as well as for materials with a high carbon content, such as organics (Khare et al. 1990; Jenniskens 1993) and amorphous carbon (Duley 1984). A common feature of this group of materials is that, in the visible, their $\operatorname{Im}(m)$ significantly and nonmonotonically decreases while $\lambda$ increases. Additionally, the strongest drop of $\operatorname{Im}(m)$ corresponds to a shift from the blue to green wavelengths. In what follows, the gradient of $\operatorname{Im}(m)$ is significantly relaxed. However, between the green and red parts of spectrum, it is still noticeable; whereas, in the red and nearinfrared parts of spectrum, $\operatorname{Im}(m)$ may experience either small variations, although not necessarily a decrease, or remain nearly constant. Thus, a species of cometary dust that belongs to the second group of materials, in the $B$ and $R$ filters, may shift to the first group of materials within the $R$ and $I$ filters. This type of wavelength dependence of $\operatorname{Im}(m)$ can produce a dramatic difference in the color slope of dust in a comet when it is measured with those two pairs of filters.

\section{Results}

For demonstration purposes, we consider the experimentally measured refractive index of $\mathrm{Mg}$-rich pyroxene $\left(\mathrm{Mg}_{0.7}\right.$ $\mathrm{Fe}_{0.3} \mathrm{SiO}_{3}$ ) from Table 4 of Dorschner et al. (1995) at three wavelengths: $m=1.649+0.0105 i(\lambda=0.45 \mu \mathrm{m}), m=1.629+0.0032 i$ $(\lambda=0.65 \mu \mathrm{m})$, and $m=1.619+0.0027 i \quad(\lambda=0.90 \mu \mathrm{m})$. This material is consistent with in situ findings in comets and the ground-based mid-IR spectrometry of comets (see, e.g., Wooden 2008 , for review). As one can see, its imaginary part decreases by a factor of 3.28 between $\lambda=0.45 \mu \mathrm{m}$ and $0.65 \mu \mathrm{m}$. However, $\operatorname{Im}(m)$ remains nearly the same between $\lambda=0.65 \mu \mathrm{m}$ and $0.9 \mu \mathrm{m}$, where the change is only a factor of $\sim 1.19$.

As a model of cometary dust particles, we use the agglomerated debris particles, whose fluffy and irregular morphology appears to be in good quantitative accordance with what was detected in situ in micron-sized dust particles in comets (see, e.g., Zubko et al. 2016; Chornaya et al. 2020). At the top of Fig. 1, we demonstrate the images of four samples of these particles. These particles have been proven capable of fitting photometric and/or polarimetric observations of comets as well as laboratory optical measurements of cometary dust analogs (see Zubko et al. 2020b, for review). In particular, the agglomerated debris particles were considered in the analysis and interpretation of photometric observations of Comet 41P/T-G-K in Luk'yanyk et al. (2019).

Using the techniques outlined by Zubko (2020), Videen et al. (2018), and Zubko et al. (2018), we calculated the lightscattering properties of agglomerated debris or pyroxene (Dorschner et al. 1995). We used the results of computations at $\lambda=0.45$ and $0.644 \mu \mathrm{m}$ to model observations in the $B$ and $R$ filters of the Johnson-Cousins photometric system and of the $R$ and $I$ filters. This analysis of multiband photometric observations of Comet 29P/Schwassmann-Wachmann 1 is similar to that of Picazzio et al. (2019). The refractive index of the agglomerated debris particles in the model $B, R$, and $I$ filters is assigned to the values adapted from Dorschner et al. (1995) at the closest wavelength, that is, $m_{B}=1.649+0.0105 i, m_{R}=1.629+0.0032 i$, and $m_{I}=1.619+0.0027 i$.

Figure 1 demonstrates the color slope $S^{\prime}$ inferred for two pairs of model filters as a function of the power index $n$ of the power-law size distribution of the agglomerated debris particles $r^{-n}$. The results, corresponding to the $B-R$ color, are shown with a solid red line and the $R-I$ color slope is shown with a blue dashed line.

The middle panel in Fig. 1 shows the results obtained at phase angle $\alpha=25^{\circ}$, which is representative of the color slope on March 3, 2017 inferred from observations by Luk'yanyk et al. (2019) and Moulane et al. (2018) (comparison was conducted by Boehnhardt et al. 2020). The bottom panel in Fig. 1 shows $S^{\prime}$ at $\alpha=70^{\circ}$, which is compliant with the comparison of observations in Moulane et al. (2018) and Boehnhardt et al. (2020) on April 12-13, 2017; such a comparison was conducted in Sect. 1 of this work. When comparing the middle and bottom panels, one can see a noticeable dependence of the color slope on the phase angle. Such a difference could be even greater when one of the phase angles approaches zero (Zubko et al. 2014). 

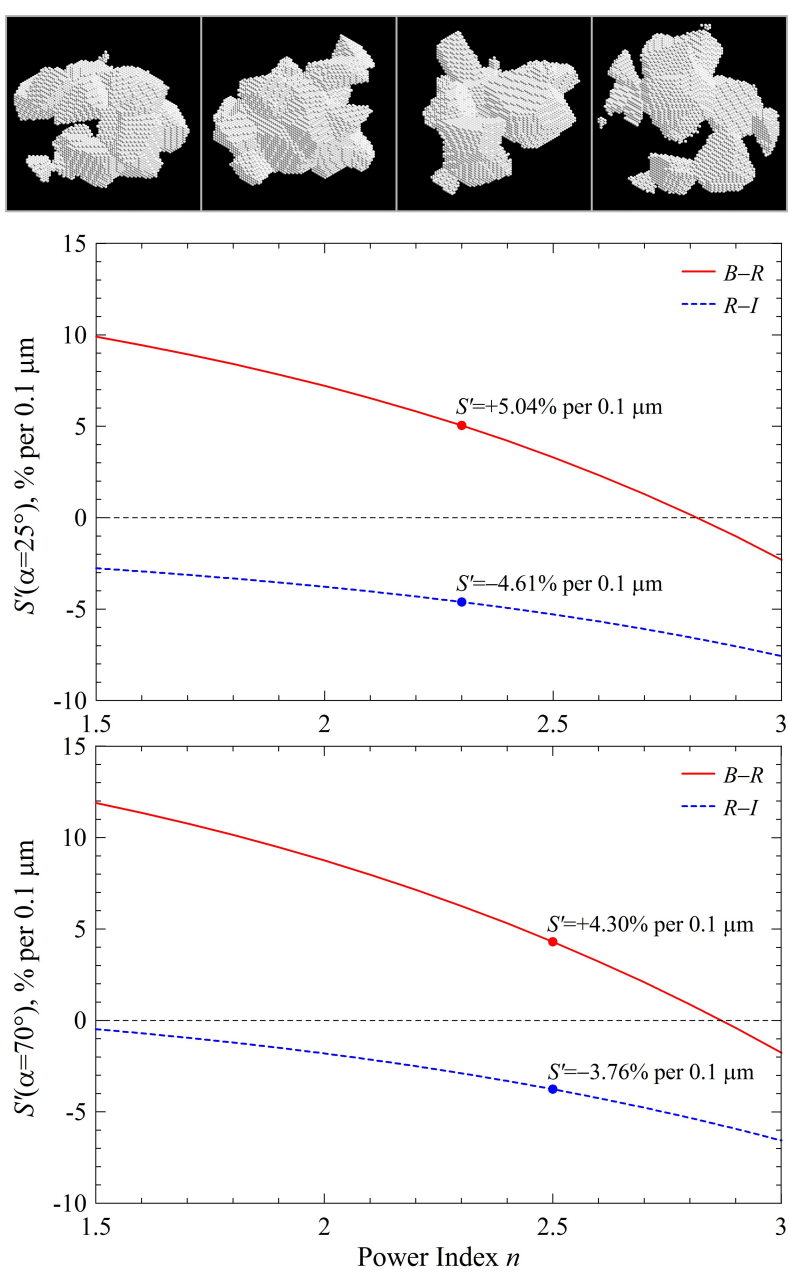

Fig. 1. Four examples of agglomerated debris particles (top) and the resulting color slope as a function of the index in a power-law size distribution $r^{-n}$ (middle and bottom) of Mg-rich pyroxene (Dorschner et al. 1995). The solid red curves correspond to the modeling of the agglomerated debris particles in the $B$ and $R$ filters; whereas, the blue dashed lines correspond to that in the $R$ and $I$ filters.

What immediately emerges from Fig. 1 is that there is a dramatic dependence of $S^{\prime}$ on the choice of filter pairs: the $B-R$ pair reveals a systematically redder color of dust compared to the $R-I$ pair. Such a difference does not suggest an observation error, but rather it reveals a complex wavelength dependence on the imaginary part of the refractive index in dust of Comet 41P/T-G-K, which, nevertheless, is consistent with what can be calculated based on measurements of various cometary-dust analogs. It is worth noting that, at some values of the power index, that is, $n=2.3$ in the middle panel and 2.5 in the bottom panel, one can obtain nearly opposite values for the color slope in the $B-R$ pair and in the $R-I$ pair.

\section{Conclusion}

Colorimetry of comets conducted with different pairs of filters may yield confusing, even seemingly contradictory results. For example, in one set of filters, a comet can be red; whereas, in another it can be blue. These types of results are not necessarily indicative of an observational error, but instead they can be the result of the particular filters used as well as the chemical composition of cometary dust particles and of different phase angles of observation. Observations of Comet 41P/TG-K by Boehnhardt et al. (2020), Luk'yanyk et al. (2019), and Moulane et al. (2018) yield seemingly contradictory colorimetry results. However, we demonstrate that these results are consistent, and they can even be modeled using pyroxene dust. Most importantly, the use of different filter sets can be used to constrain the chemical properties of cometary dust.

Acknowledgements. The authors thank two anonymous referees for their constructive reviews.

\section{References}

A'Hearn, M. F., Schleicher, D. G., Millis, R. L., Feldman, P. D., \& Thompson, D. T. 1984 , AJ, 89, 579

A'Hearn, M. F., Millis, R. C., Schleicher, D. O., et al. 1995, Icarus, 118, 223

A'Hearn, M. F., Belton, M. J. S., Delamere, W. A., et al. 2011, Science, 332, 1396

Boehnhardt, H., Riffeser, A., Ries, C., et al. 2020, A\&A, 638, A8

Chornaya, E., Zubko, E., Luk'yanyk, I., et al. 2020, Icarus, 337, 113471

Chubko, L. S., Churyumov, K. I., Afanasiev, V. L., et al. 2009, Deep Impact as a

World Observatory Event: Synergies in Space, Time, and Wavelength, 197

Clairemidi, J., Moreels, G., \& Krasnopolsky, V. A. 1990, Icarus, 86, 115

Combi, M. R., Harris, W. M., \& Smyth, W. H. 2004, in Comets II, eds. M. C.

Festou, H. U. Keller, \& H. A. Weaver (Tucson: University of Arizona Press), 523

Dorschner, J., Begemann, B., Henning, T., et al. 1995, A\&A, 300, 503

Duley, W. W. 1984, ApJ, 287, 694

Farnham, T. L., Schleicher, D. G., \& A'Hearn, M. F. 2000, Icarus, 147, 180

Fink, U., Combi, M. R., \& Disanti, M. A. 1991, ApJ, 383, 356

Ivanova, O., Rosenbush, V., Afanasiev, V., et al. 2017a, Icarus, 284, 167

Ivanova, O., Zubko, E., Videen, G., et al. 2017b, MNRAS, 469, 2695

Jackson, W. M., Bao, Y., \& Urdahl, R. S. 1991, J. Geophys. Res., 96, 17569

Jenniskens, P. 1993, A\&A, 274, 653

Jewitt, D. C., Soifer, B. T., Neugebauer, G., et al. 1982, AJ, 87, 1854

Khare, B. N., Thompson, W. R., Sagan, C., et al. 1990, Lunar and Planetary Science Conference, 21, 627

Khare, B. N., Thompson, W. R., Cheng, L., et al. 1993, Icarus, 103, 290

Lamy, P. L., Toth, I., Weaver, H. A., A’Hearn, M. F., \& Jorda, L. 2011, MNRAS, 412, 1573

Luk’yanyk, I., Zubko, E., Husárik, M., et al. 2019, MNRAS, 485, 4013

Mazets, E. P., Aptekar, R. L., Golenetskii, S. V., et al. 1986, Nature, 321, 276

Moulane, Y., Jehin, E., Opitom, C., et al. 2018, A\&A, 619, A156

Picazzio, E., Luk'yanyk, I. V., Ivanova, O. V., et al. 2019, Icarus, 319, 58

Price, M. C., Kearsley, A. T., Burchell, M. J., et al. 2010, Meteor. Planet. Sci., 45, 1409

Rosenbush, V., Ivanova, O., Kleshchonok, V., et al. 2020, Icarus, 348, 113767

Rousselot, P., Clairemidi, J., \& Moreels, G. 1994, A\&A, 286, 645

Videen, G., Zubko, E., Arnold, J. A., et al. 2018, J. Quant. Spectr. Rad. Transf., 211,123

Virkki, A., Zubko, E., Nolan, M. C., et al. 2019, Icarus, 325, 94

Warren, S. G. 1984, Appl. Opt., 23, 1206

Wooden, D. H. 2008, Space Sci. Rev., 138, 75

Zubko, E. 2020, MNRAS, 492, 810

Zubko, E., Muinonen, K., Videen, G., et al. 2014, MNRAS, 440, 2928

Zubko, E., Shkuratov, Y., \& Videen, G. 2015a, J. Quant. Spectr. Radiat. Transf., 150,42

Zubko, E., Videen, G., Hines, D. C., et al. 2015b, Planet. Space Sci., 118, 138

Zubko, E., Videen, G., Hines, D. C., et al. 2016, Planet. Space Sci., 123, 63

Zubko, E., Videen, G., Arnold, J. A., et al. 2018, Opt. Lett., 43, 4308

Zubko, E., Chornaya, E., Zheltobryukhov, M., et al. 2020a, Icarus, 336, 113453

Zubko, E., Videen, G., Arnold, J. A., et al. 2020b, ApJ, 895, 110 